\title{
Burnout among German oncologists: a cross-sectional study in cooperation with the Arbeitsgemeinschaft Internistische Onkologie Quality of Life Working Group
}

\author{
Madeleine Helaß ${ }^{1}\left(\mathbb{0} \cdot\right.$ Georg Martin Haag ${ }^{2}\left(\mathbb{D} \cdot\right.$ Ulli Simone Bankstahl ${ }^{3} \cdot$ Deniz Gencer $^{4} \cdot$ Imad Maatouk $^{1,5,6}(\mathbb{D}$
}

Received: 9 November 2021 / Accepted: 29 January 2022 / Published online: 13 February 2022

(c) The Author(s) 2022

\begin{abstract}
Purpose Oncologists are at an increased risk of developing burnout, leading to negative consequences in patient care and in professional satisfaction and quality of life. This study was designed to investigate exhaustion and disengagement among German oncologists and assess the prevalence of burnout among oncologists within different professional settings. Furthermore, we wanted to examine possible relations between sociodemographic factors, the oncological setting, professional experience and different aspects of burnout.

Methods In a cross-sectional study design, an Internet-based survey was conducted with 121 oncologists between April and July 2020 using the Oldenburg Burnout Inventory, which contains items on exhaustion, disengagement, and burnout. Furthermore, sociodemographic data of the participants were assessed. The participants were members of the Working Group Medical Oncology (Arbeitsgemeinschaft Internistische Onkologie) within the German Cancer Society.

Results The survey showed a burnout prevalence of $43.8 \%$, which correlated with age and professional experience; that is, the prevalence is particularly high among younger oncologists. Exhaustion is closely related to employment status; that is, it was significantly higher among employed oncologists. There were remarkably low levels of disengagement among oncologists, highlighting the own demand to fulfil job requirements despite imminent or actual overburdening in daily work. Conclusion More support is necessary to mitigate the professional stressors in the healthcare system. To ensure quality medical care, employees should be offered preventive mental health services early in their careers.
\end{abstract}

Keywords Burnout $\cdot$ Exhaustion $\cdot$ Disengagement $\cdot$ Oldenburg burnout inventory $\cdot$ Oncologist $\cdot$ Prevalence

\section{Introduction}

Since the second half of the last century, burnout among healthcare workers in hospitals has been becoming the focus of health psychology and occupational medicine

Madeleine Helaß and Georg Martin Haag share first authorship.

Imad Maatouk

Maatouk_I@ukw.de

1 Department of General Internal Medicine and Psychosomatics, Heidelberg University Hospital, Heidelberg, Germany

2 Department of Medical Oncology, National Centre for Tumour Diseases (NCT), Heidelberg University Hospital, Heidelberg, Germany

3 Institute for Clinical-Oncological Research (IKF), Krankenhaus Nordwest gGmbH, Frankfurt, Germany research. Compared with the general population, health care professionals are at an increased risk of developing symptoms of burnout (Shanafelt et al. 2012; Dyrbye et al. 2014). Maslach's fundamental work defined burnout as work-related stress in terms of three components: emotional exhaustion (EE), depersonalization (DP) and low personal accomplishment (PA). Moreover, to date, the

4 Department of Medicine III, Medical Faculty Mannheim, University Medical Center Mannheim, Heidelberg University, Heidelberg, Germany

5 Section of Psychosomatic Medicine, Psychotherapy and Psycho-Oncology, Department of Internal Medicine II, Julius-Maximilian University Würzburg, Würzburg, Germany

6 Psychosomatics, Psychotherapy and Psychooncology, University Hospital Würzburg, Medical Clinic II, Oberdürrbacher Str. 6, 97080 Würzburg, Germany 
Maslach Burnout Inventory (MBI) (Maslach et al. 1981) is the most widely used and accepted survey to assess burnout (Rotenstein et al. 2018).

During the last 20 years, an increasing prevalence of burnout among physicians has been observed (Linzer et al. 2001; Eelen et al. 2014; Shanafelt et al. 2015), though the prevalence rates vary significantly between different surveys depending on the analyzed diagnostic criteria, demographic factors and medical discipline (Prins et al. 2007; Yates \& Samuel 2019). The highest rates were observed in emergency care, neurology and general internal medicine (Shanafelt et al. 2012, 2015), especially among oncologists (Yates and Samuel 2019).

Several factors have been identified as risk factors for physicians developing burnout, including workload (Cooper et al. 1989; Freeborn 2001). Among these factors are inefficient work processes (Shanafelt, et al. 2016a, b), time pressure (Kleiner and Wallace 2017) and administrational issues (Sinsky et al. 2016). Beyond that, oncologists are often confronted with patients deteriorating significantly or dying due to an advancing tumor disease, despite their efforts in terms of oncological care (Trufelli et al. 2008), focusing not only on prolongation of survival but also on improving or maintaining the patient's quality of life. Thus, apart from tumor-specific therapy, concomitant supportive treatment is integral part of oncologist's daily work to reach this goal.

Burnout among physicians can have devastating effects on patient care, including a significantly increased risk of medical errors (Shanafelt et al. 2010; Hall et al. 2016), a decreased job productivity (Dewa et al. 2014; Shanafelt et al. 2016) and a lower patient satisfaction rate (Haas et al. 2000; Halbesleben and Rathert 2008). Furthermore, physicians with burnout may suffer from somatic and mental health problems, including depression and substance abuse (Oreskovich et al. 2012; Medisauskaite and Kamau 2017), an increased risk of vehicular accidents (West et al. 2012) and an increased suicide risk (van der Heijden et al. 2008). Hence, the assessment of the prevalence of burnout among clinically active oncologists is of great interest to researchers, clinicians, and policy makers.

The prevalence of burnout repeatedly leads to debates (Banerjee et al. 2017; Bianchi et al. 2017), and the MBI (Maslach et al. 1981) has revealed some psychometric weaknesses (i.e. factor validity and one-sided wording of items) (for further information, see Demerouti et al. 2003; Demerouti and Bakker 2008). A verification of the prevalence via repetitive measurements of the burnout diagnostic criteria seems to be necessary. Therefore, this study was designed to investigate two dimensions of burnout using a well-validated questionnaire in a German sample of oncologists working in different oncological settings. Furthermore, we wanted to examine possible relations between sociodemographic factors, the oncological setting, and work experience and different aspects of burnout.

\section{Materials and methods}

\section{Study population}

All members of the Working Group Medical Oncology (Arbeitsgemeinschaft Internistische Onkologie (AIO)) within the German Cancer Society were asked via email to participate in an Internet-based survey. The inclusion criteria were a minimum age of 18 years and a medical practice with patients with cancer. This cross-sectional study, platform provided by SoSci Survey, was open for 3 months from April to July in 2020. The access was only possible using a hyperlink sent in an email circular sent to all AIO members. Participation in the survey was voluntary and anonymous. The manuscript was prepared according to the Strengthening the Reporting of Observational Studies in Epidemiology statement criteria (Von Elm et al. 2007).

\section{Questionnaire}

The 16-item Oldenburg Burnout Inventory (OLBI) (Demerouti and Bakker 2008) was assessed to measure the two following subscales-exhaustion and disengagement (8 items per subscale) - on a 5-point Likert scale ( $1=$ "strongly disagree" to $5=$ "strongly agree"). Four items of each subscale were inverted. The exhaustion dimension $\left(\mathrm{OLBI}_{\mathrm{exh}}\right)$ refers to the "general feelings of emptiness, overtaxing from work, a strong need for rest and a state of physical exhaustion" (Demerouti and Bakker 2008, p. 10). Exhaustion refers to the long-term consequence of intense physical, affective, and cognitive stress, while emotional exhaustion $\left(\mathrm{MBI}_{\mathrm{EE}}\right)$ surveys only affective aspects. Depersonalization $\left(\mathrm{MBI}_{\mathrm{DP}}\right)$ refers to distancing from a beneficiary and is only one aspect of the disengagement scale $\left(\mathrm{OLBI}_{\mathrm{dis}}\right)$. OLBI $\mathrm{dis}_{\mathrm{dis}}$ refers to "distancing oneself from one's work and the associated attitudes and attitudes toward work, to negative cynical attitudes and behaviors toward one's work in general" (ibid., p. 10). The last issue in particular seemed crucial, as work-related attitudes are predictors of job performance (Hettiararchchi and Jayarathna 2014), and job involvement is related to job satisfaction (Awadh and Wan Ismail 2012).

Both subscales had a reliability of 0.85 . Bivariate correlations between both subscales were $0.55(p<0.01)$ for healthcare workers with mean levels of exhaustion $(M=2.53)$ and disengagement $(M=2.38)$ (Demerouti and Bakker 2008). Since there is no standard cutoff for burnout in the OLBI, we first used $\mathrm{M}_{\mathrm{OLBI}} \geq 2.18$ as the mean value of the disengagement and exhaustion scales (Peter Chernoff et al. 2019). Further analyses are limited to the more sensitive cutoff of 
$\mathrm{M}_{\mathrm{Exh}} \geq 2.5$ (Block et al. 2020) for the exhaustion subscale as a "burnout measure."

Furthermore, sociodemographic data, profession, medical field, professional experience, workplace, and employment status, board certification, work on inpatient palliative care unit (PCU) and participation in an outpatient palliative care team (Spezialisierte Ambulante Palliativversorgung (SAPV)) were assessed, including the estimated proportion of total working time, proportion of inpatient work, working hours with tumor patients and the estimated proportion of working time with palliative patients.

\section{Statistics}

To evaluate exhaustion, disengagement, and burnout among German oncologists, absolute and relative frequencies for categorical data, mean values, standard deviation, and range for continuous variables were calculated. Participants whose data were incomplete were nevertheless included, as the missing data only included some sociodemographic data. First, we used one-way analysis of variance (ANOVA) as an alternative to $t$ test to examine differences in the subgroups of sociodemographic variables in exhaustion, disengagement, and burnout. Second, correlation coefficients were measured among all study variables to examine possible relationships between them. Third, multiple regression analyses were performed between disengagement and exhaustion as dependent variables and the sociodemographic factors as independent variables to examine possible predictors for the OLBI measures. The risk ratio (RR) was used to determine the risk of burnout. For all tests, p-values of less than 0.05 were used to indicate statistical significance. Analyses were done using Statistical Package for the Social Sciences, version 27 (IBM, 2020).

Informed consent was obtained from all participants. Ethical Approval was received on September 2019 from the Ethical Committee of the Medical Faculty in Heidelberg (S-615/2019). The study was registered to the German Clinical Trials Register (DRKS500018851).

\section{Results}

\section{Characteristics of participants}

Approximately 1400 physicians were contacted, among whom 121 participated, which corresponds to a response rate of $8.64 \%$. The mean age was $50.28 \pm 9.32$ years (range, 28-75 years). This corresponds to the distribution of all age groups of AIO members (40-60 years, 49.14\%) and represents the biggest age cohort of physicians, board certified in internal medicine in Germany (40-60 years, 59.8\%) (Bundesärztekammer 2020). The sample was predominantly male ( $n=80,66 \%)$, reflecting the majority of AIO members (male 77, 59\%) as well as the majority of German oncologists (male 59.66\%). Professional experience varied widely between 2 and 49 years (median $=20-24$ years $(26 \%$, interquartile range $(\mathrm{IQR})=15-29$ years). Moreover, $113(94.9 \%)$ physicians had a board certification in hemato-oncology. The participants worked on average $82.40 \% \pm 17.13 \%$ (range, $21 \%-100 \%$ ) of their time with patients with tumor. Twentyone $(17.4 \%)$ physicians worked within a PCU, spending $27.81 \% \pm 24.11 \%$ (range $6-81 \%$ ) of their working time, whereas $11(9.1 \%)$ physicians participated in an outpatient palliative care team $(S A P V)$, accounting for $11.64 \% \pm 8.51 \%$ (range 2-34\%) of their working time. Absolute and relative frequencies are shown in Table 1 . Table 2 presents the mean values, standard deviations, ranges, and correlation coefficients. Risk ratios are shown in Table 3.

\section{Disengagement}

The mean value on the disengagement scale was $1.65 \pm 0.45$ (range, 1.00-2.88). To evaluate the disengagement scale, the range of values was divided into three parts and designated as low $(<1.63)$, medium $(1.63-2.24)$ and high $(>2.24)$. Moreover, 60 (49.59\%) physicians showed low disengagement $($ mean $=1.28 \pm 0.153$; range $=1-1.5), 46(38.02 \%)$ showed medium disengagement (mean $=1.85 \pm 0.19$; range 1.63-2.13) and $15(12.40 \%)$ showed high disengagement (mean $=2.49 \pm 0.21$; range 2.38-2.88). High disengagement was mainly related to men $(n=12,9.9 \%)$ and hospital employees ( $n=10,8.3 \%)$ (Fig. 1).

Correlation analyses, ANOVA, and regression analyses showed no significant correlations between the disengagement scale and other variables.

\section{Exhaustion}

Based on a cutoff of $\mathrm{M}_{\mathrm{OLBI}}=2.18,45$ physicians (52.3\%) of the total sample had burnout. Focusing on the more specific exhaustion scale with a cutoff of $\mathrm{M}_{\mathrm{Exh}}>2.5,53$ (43.8\%) physicians showed increased values in the exhaustion scale as a hint for suffering from burnout.

Pearson's correlation coefficients showed a significant negative correlation between age and exhaustion ( $r=-0.183, p<0.01)$; that is, the older the physician, the less exhaustion. Using a cutoff at the highest quartile (P75), physicians older than 57 years $(n=31)$ have significantly lower values (mean $=2.09 \pm 0.94$ ) on the exhaustion scale than physicians younger than 57 years $(n=90$; mean $=2.41 \pm 0.57)(t(119)=-2,581 ; p<0.05)$.

A significantly strong positive correlation was observed between age and professional experience $(r=0.830$; $p<001)$. Kendall's Tau showed a significant negative correlation between professional experience and exhaustion 


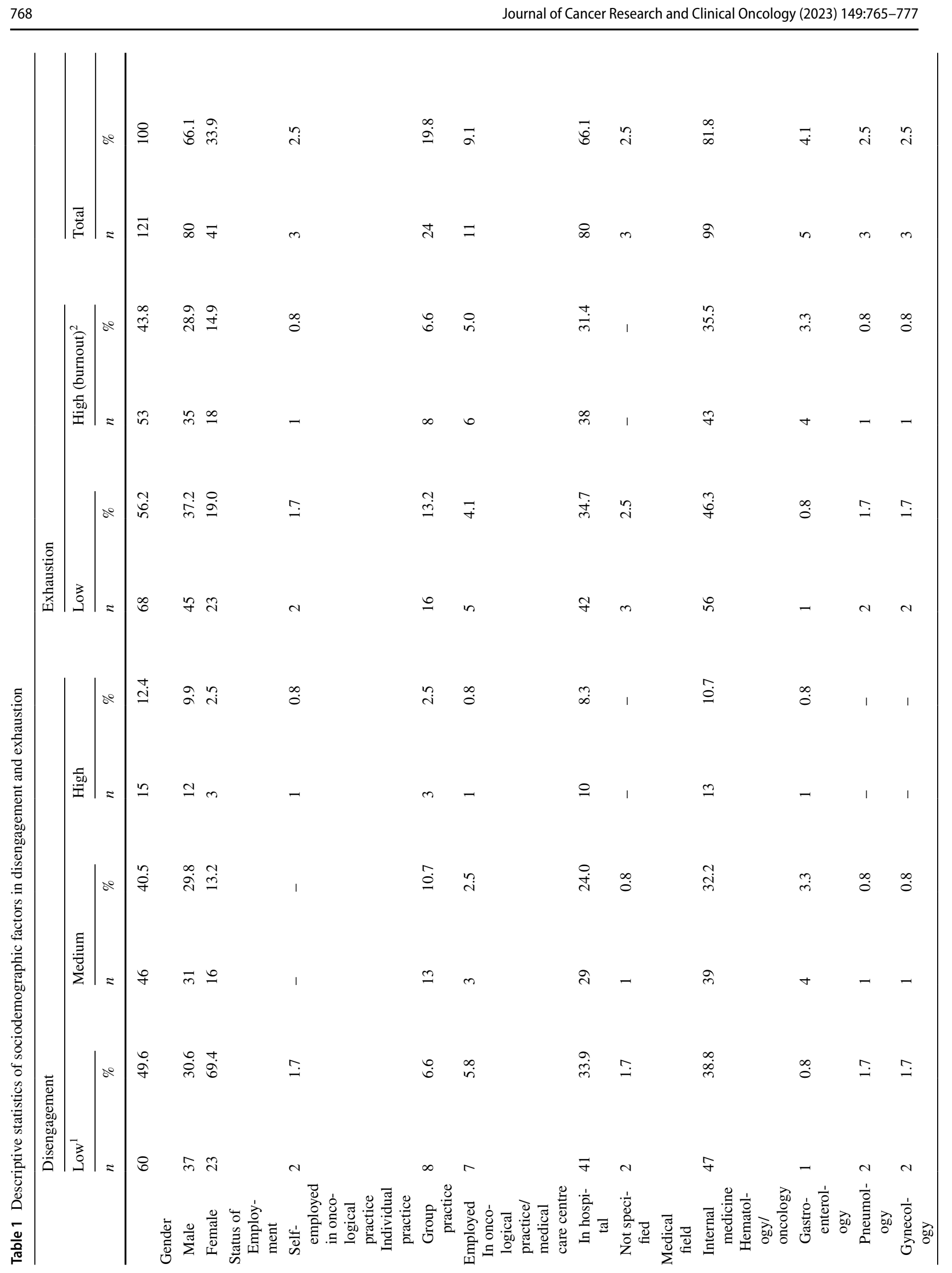

Springer 


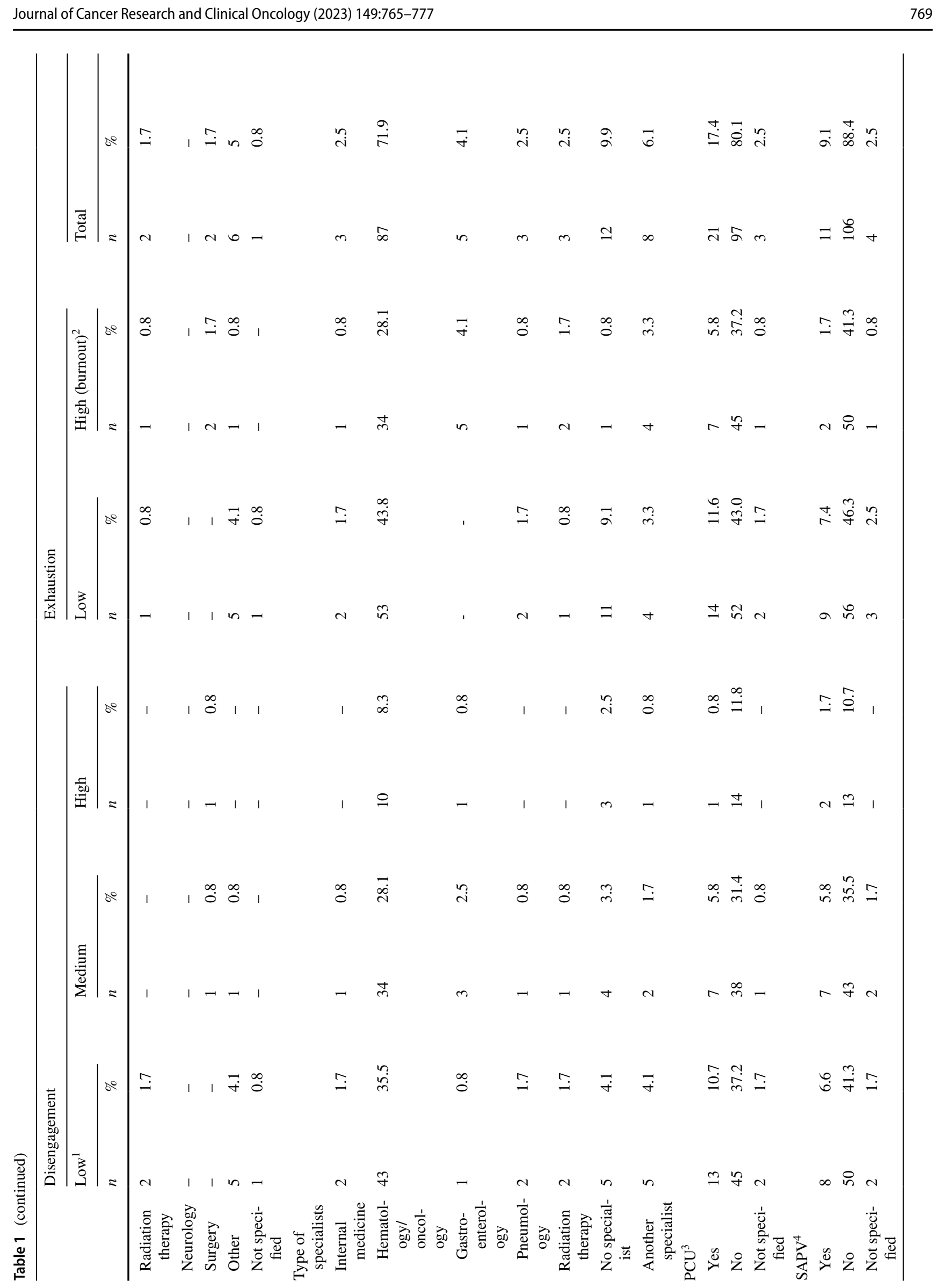

望 Springer 


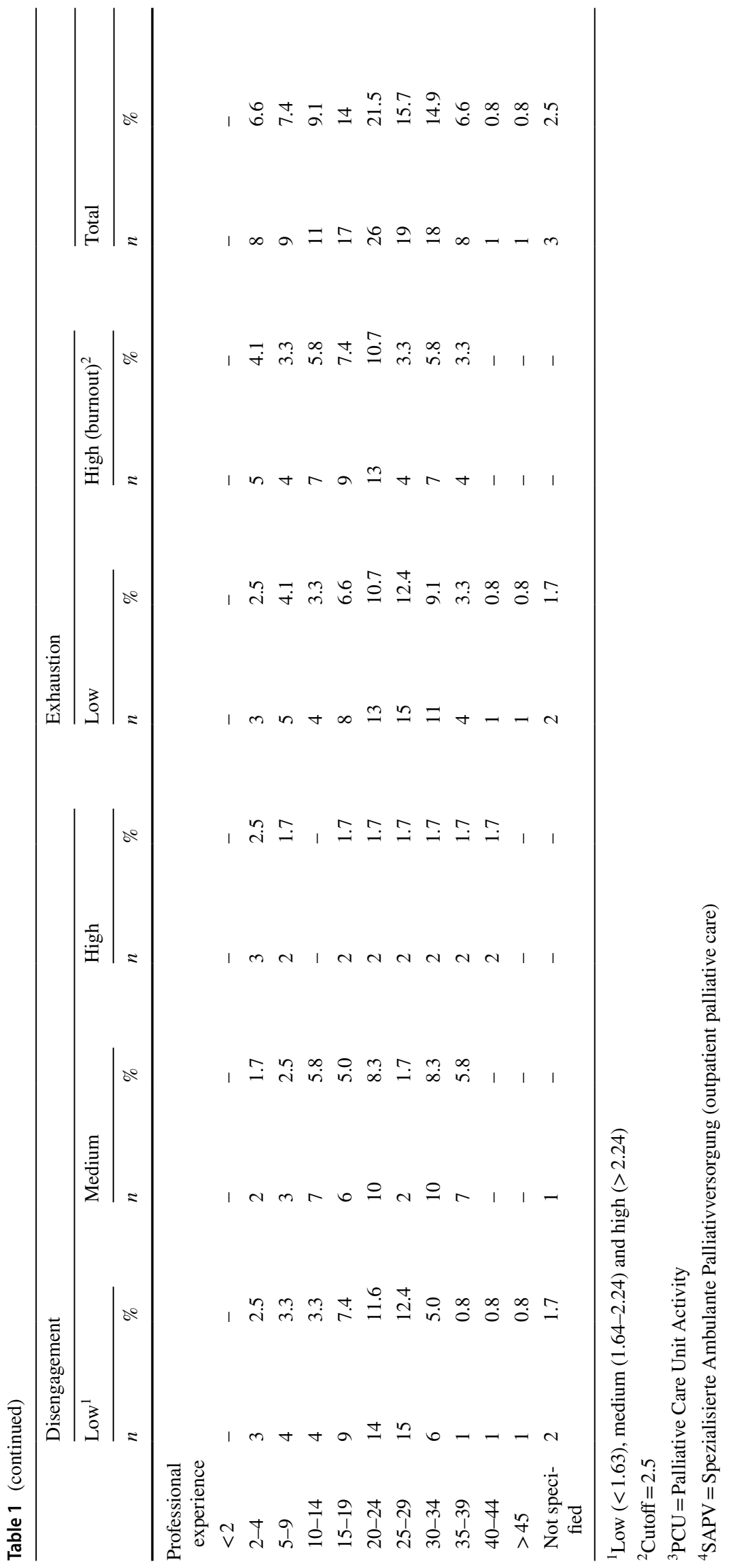




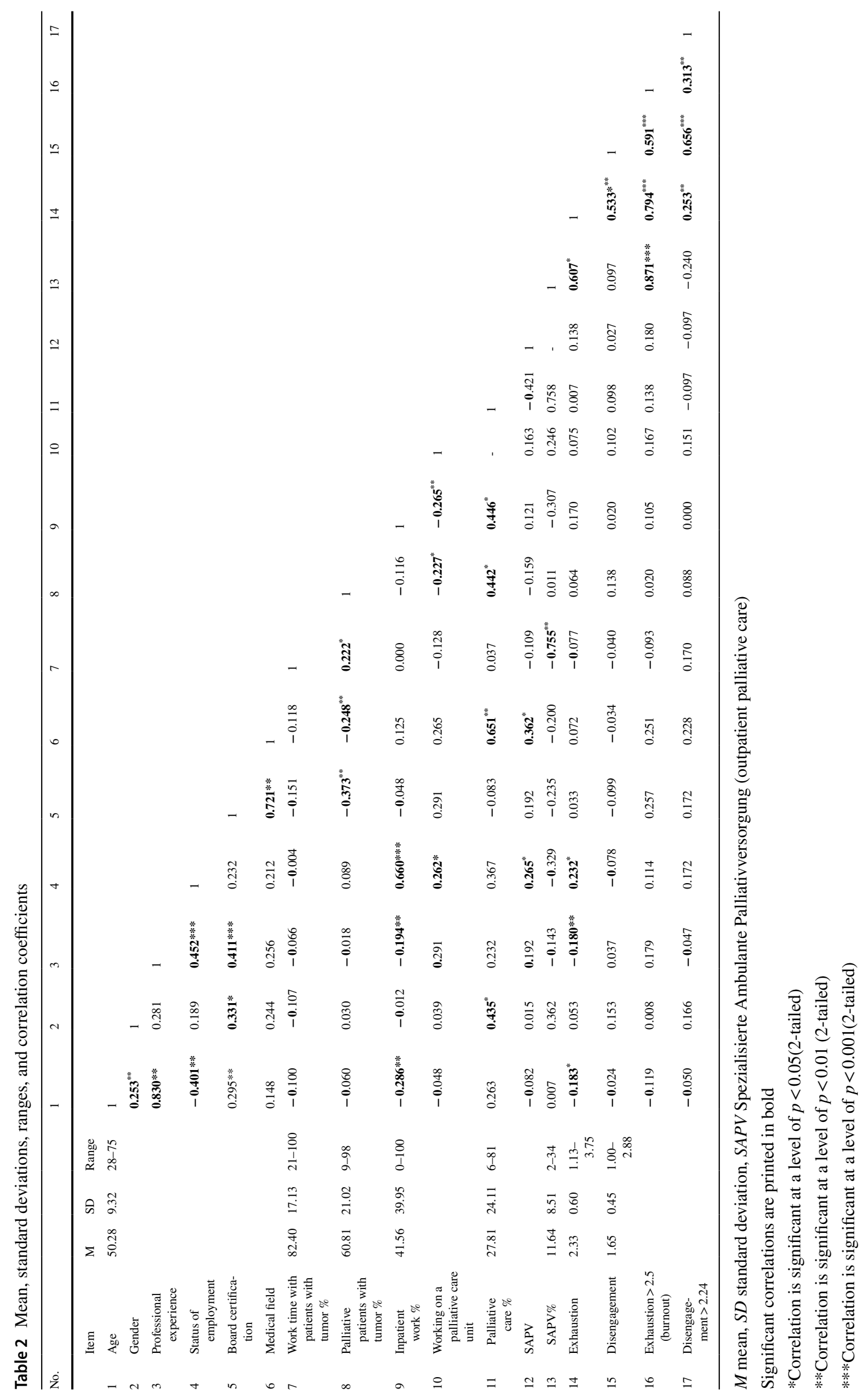


Table 3 Relative ratio for burnout among German oncologists

\begin{tabular}{|c|c|c|c|c|c|c|c|}
\hline & Cutoff/ Categories & Value & $N=53$ & $\%$ & $\mathrm{RR}[95 \% \mathrm{CI}]$ & $\begin{array}{l}\text { Pearson } \\
\text {-Chi }\end{array}$ & $p^{1}$ \\
\hline \multirow[t]{6}{*}{ Age (years) } & \multirow[t]{2}{*}{$\mathrm{P} 25=43$} & $<43$ & 14 & 50.0 & $1.192[0.767-1.853]$ & \multirow[t]{2}{*}{0.569} & \multirow[t]{2}{*}{0.451} \\
\hline & & $>43$ & 39 & 41.9 & $0.839[0.540-1.303]$ & & \\
\hline & \multirow[t]{2}{*}{$\mathrm{P} 50=52$} & $<52$ & 33 & 55.0 & $1.618[1.096-2.568]$ & \multirow[t]{2}{*}{6.063} & \multirow[t]{2}{*}{0.014} \\
\hline & & $>52$ & 20 & 32.8 & $0.596[0.389-0.913]$ & & \\
\hline & \multirow[t]{2}{*}{$\mathrm{P} 75=57$} & $<57$ & 42 & 46.7 & $1.315[0.779-2.220]$ & \multirow[t]{2}{*}{1.171} & \multirow[t]{2}{*}{0.278} \\
\hline & & $>57$ & 11 & 35.5 & $0.760[0.450-1.283]$ & & \\
\hline \multirow[t]{6}{*}{ Work time with patients with tumor (\%) } & \multirow[t]{2}{*}{$\mathrm{P} 25=76$} & $<76$ & 15 & 55.6 & $1.330[0.878-2.016]$ & \multirow[t]{2}{*}{1.602} & \multirow[t]{2}{*}{0.206} \\
\hline & & $\geq 76$ & 38 & 41.8 & $0.752[0.496-1.139]$ & & \\
\hline & \multirow[t]{2}{*}{$\mathrm{P} 50=86$} & $<86$ & 29 & 51.8 & $1.338[0.895-2.001]$ & \multirow[t]{2}{*}{2.033} & \multirow[t]{2}{*}{0.154} \\
\hline & & $\geq 86$ & 24 & 38.7 & $0.747[0.500-1.118]$ & & \\
\hline & \multirow[t]{2}{*}{$\mathrm{P} 75=96$} & $<96$ & 41 & 47.7 & $1.271[0.772-2.094]$ & \multirow[t]{2}{*}{0.976} & \multirow[t]{2}{*}{0.323} \\
\hline & & $\geq 96$ & 12 & 37.5 & $0.787[0.478-1.290]$ & & \\
\hline Palliative patients with tumor (\%) & $\mathrm{P} 25=48$ & $<48$ & 11 & 39.3 & $0.842[0.565-1.403]$ & 0.470 & 0.493 \\
\hline & & $\geq 48$ & 42 & 46.7 & $1.188[0.713-1.980]$ & & \\
\hline & $\mathrm{P} 50=65$ & $<65$ & 26 & 44.8 & $0.996[0.668-1.486]$ & 0.000 & 0.985 \\
\hline & & $\geq 65$ & 27 & 45.0 & $1.004[0.673-1.497]$ & & \\
\hline & $\mathrm{P} 75=78.25$ & $<78.25$ & 41 & 46.1 & $1.113[0.683-1.814]$ & 0.194 & 0.659 \\
\hline & & $\geq 78.25$ & 12 & 41.4 & $0.898[0.551-1.463]$ & & \\
\hline Inpatients $(\%)$ & $\mathrm{P} 25=6$ & $<6$ & 13 & 52.0 & $1.183[0.760-1.841]$ & 0.511 & 0.475 \\
\hline & & $\geq 6$ & 40 & 44.0 & $0.845[0.543-1.313]$ & & \\
\hline & $\mathrm{P} 50=34,5$ & $<34.5$ & 26 & 44.8 & $0.963[0.647-1.432]$ & 0.035 & 0.852 \\
\hline & & $\geq 34.5$ & 27 & 46.6 & 1.038 [0.698-1.544] & & \\
\hline & $\mathrm{P} 75=76.75$ & $<76.75$ & 39 & 44.8 & $0.929[0.596-1.446\}$ & 0.104 & 0.747 \\
\hline & & $\geq 76.75$ & 14 & 48.3 & 1.077 [0.691-1.677] & & \\
\hline $\mathrm{PCU}^{2}(\%)$ & $\mathrm{P} 25=7$ & $<7$ & 2 & 40.0 & $1.280[0.350-4.680]$ & 0.131 & 0.717 \\
\hline & & $\geq 7$ & 5 & 31.3 & $0.781[0.214-2.850]$ & & \\
\hline & $\mathrm{P} 50=18$ & $<18$ & 5 & 50.0 & 2.750 [0.679-11.134] & 2.386 & 0.122 \\
\hline & & $\geq 18$ & 2 & 18.2 & $0.364[0.090-1.472]$ & & \\
\hline & $\mathrm{P} 75=43$ & $<43$ & 5 & 31.3 & $0.787[0.214-2.856]$ & 0.131 & 0.717 \\
\hline & & $\geq 43$ & 2 & 40.0 & $1.280[0.350-4.680]$ & & \\
\hline $\operatorname{SAPV}^{3}(\%)$ & $\mathrm{P} 25=6$ & $<6$ & 1 & 50.0 & $4.500[0.447-45.328]$ & 1.664 & 0.197 \\
\hline & & $\geq 6$ & 1 & 11.0 & $0.222[0.022-2.238]$ & & \\
\hline & $\mathrm{P} 50=11$ & $<11$ & 1 & 20.0 & $0.835[0.068-10.20]$ & 0.020 & 0.887 \\
\hline & & $\geq 11$ & 1 & 16.7 & $1.200[0.098-14.69]$ & & \\
\hline & $\mathrm{P} 75=12$ & $<12$ & 1 & 12.5 & $0.375[0.033-4.275]$ & 0.637 & 0.425 \\
\hline & & $\geq 12$ & 1 & 33.3 & 2.667 [0.234-30.399] & & \\
\hline Professional experience (years) & $\mathrm{P} 25=15-19$ & $<15$ & 16 & 57.1 & $1.390[0.927-2.084]$ & 2.218 & 0.139 \\
\hline & & $\geq 15$ & 37 & 41.1 & $0.719[0.480-1.079]$ & & \\
\hline & $\mathrm{P} 50=20-24$ & $<20$ & 25 & 55.6 & $1.448[0.9800-2.141]$ & 3.329 & 0.068 \\
\hline & & $\geq 20$ & 28 & 38.4 & $0.690[0.467-1.021]$ & & \\
\hline & $P 75=25-29$ & $<25$ & 38 & 53.5 & $1.677[1.048-2.684]$ & 5.336 & 0.021 \\
\hline & & $\geq 25$ & 15 & 31.9 & $0.596[0.373-0.955]$ & & \\
\hline Gender & Male & & 35 & 43.9 & $0.997[0.715-1.391]$ & 0.000 & 0.987 \\
\hline & Female & & 18 & 43.8 & $1.003[0.655-1.536]$ & & \\
\hline PCU & Yes & & 7 & 33.6 & $7.11[0.372-1.350]$ & 1.280 & 0.258 \\
\hline & No & & 45 & 46.9 & $1.255[0.879-1.372]$ & & \\
\hline SAPV & Yes & & 2 & 18.2 & $0.385[0.108-1.372]$ & 3.392 & 0.660 \\
\hline & No & & 50 & 47.2 & $1.549[1.112-2.158]$ & & \\
\hline Status of employment & Self-employed & & 9 & 33.3 & $0.613[0.301-1.251]$ & 1.898 & 0.168 \\
\hline
\end{tabular}


Table 3 (continued)

\begin{tabular}{|c|c|c|c|c|c|c|c|}
\hline & Cutoff/ Categories & Value & $N=53$ & $\%$ & $\mathrm{RR}[95 \% \mathrm{CI}]$ & $\begin{array}{l}\text { Pearson } \\
\text {-Chi }\end{array}$ & $p^{1}$ \\
\hline \multirow{3}{*}{ Board certification } & Employed & & 44 & 48.8 & 1.148 [0.949-1.393] & \multirow{3}{*}{0.208} & \multirow{3}{*}{0.648} \\
\hline & Yes & & 47 & 43.1 & 0.973 [0.861-1.089] & & \\
\hline & No & & 6 & 50.0 & $1.283[0.439-3.752]$ & & \\
\hline \multirow{2}{*}{$\begin{array}{l}\text { Board certification internal medicine: hematology/ } \\
\text { oncology }\end{array}$} & Yes & & 34 & 39.1 & 0.823 [0.649-1.044] & \multirow[t]{2}{*}{2.804} & \multirow[t]{2}{*}{0.094} \\
\hline & No & & 19 & 55.9 & 1.635 [0.916-2.885] & & \\
\hline \multirow{2}{*}{$\begin{array}{l}\text { Medical field: Internal medicine: Hematology/oncol- } \\
\text { ogy }\end{array}$} & Yes & & 43 & 43.4 & $0.971[.821-1.148$ & \multirow[t]{2}{*}{0.123} & \multirow[t]{2}{*}{0.726} \\
\hline & No & & 10 & 47.6 & $1.149[.528-2.499]$ & & \\
\hline
\end{tabular}

Statistically significant differences are printed in bold

${ }^{1}$ Significant at a level of $p<0.05$ (2-tailed)

${ }^{2} \mathrm{PCU}=$ Palliative Care Unit Activity

${ }^{3} \mathrm{SAPV}=$ Spezialisierte Ambulante Palliativversorgung (outpatient palliative care)

$(r=-0.180 ; p<0.01)$; that is, the less work experience, the greater the exhaustion. Pearson's chi-square test confirmed an association between professional experience of less than 20 years with burnout $\left(\mathrm{M}_{\mathrm{Exh}} \geq 2.5\right): \chi^{2}(1)=5.176$; $p<0.05, \varphi=0.209$. The risk of burnout among physicians with a professional experience of less than 25 years $(n=38$, $53.5 \%$ ) was thrice higher than that among physicians with a professional experience of at least 25 years $(n=25,31.9 \%)$ $(\mathrm{RR}=1.677,95 \%$ CI 1.129-6.419; $p<0.05)$ (Fig. 2).

Pearson's correlation coefficients showed a slightly positive correlation between employment status and exhaustion $(r=0.232 ; p<0.05)$. The difference in exhaustion between employed (mean $=2.415 \pm 0.582$ ) and selfemployed (mean $=2.08 \pm 0.60)$ physicians was significant $(t(119)=2.576 ; p<0.05)$, suggesting that employed physicians describe themselves as more exhausted than

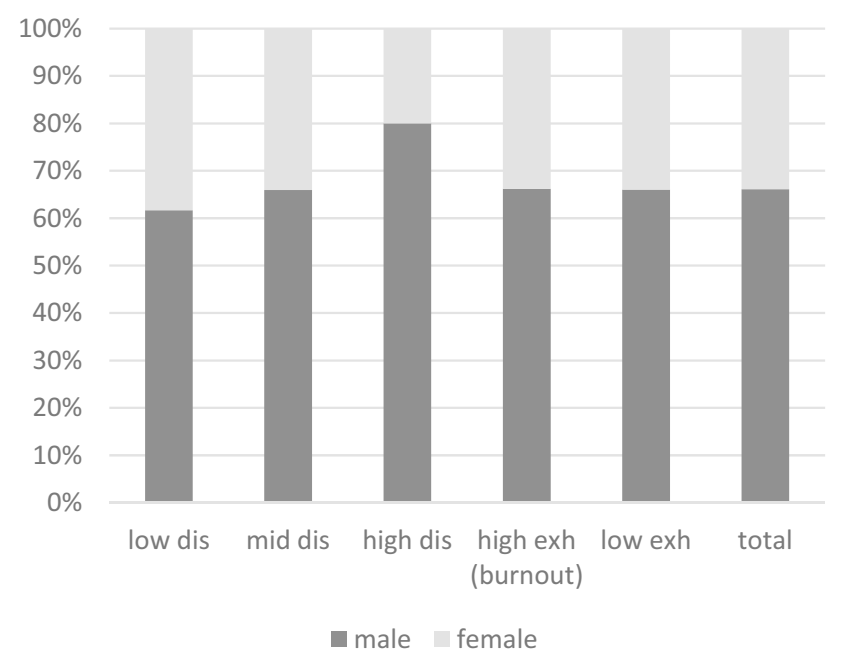

Fig. 1 Gender distribution of disengagement (i.e. low, middle and high) and exhaustion (i.e. low and high [burnout]) self-employed colleagues. The comparison of the mean values of exhaustion showed a significant difference $(t(114)=2.115 ; p<0.05)$ between physicians treating less than $30 \%$ of inpatients $(n=55$; mean $=2.22 \pm 0.593)$ and physicians treating more than $30 \%$ of inpatients (mean $=2.45 \pm 0.609)$. Moreover, $35(32.4 \%)$ employed and seven $(9.6 \%)$ self-employed physicians showed signs of burnout; the relative risk was insignificant.

Physicians participating in an outpatient palliative care team (SAPV) were predominantly male $(n=7,63.6 \%)$ with a mean age of $52.82 \pm 5.741$ years (range $41-60$ years) and a professional experience between 5 and 34 years (median $=20-24$ years $[27.3 \%, I Q R=15-29$ years $]$ ). They were predominantly hemato-oncology specialists $(n=9$, $81.8 \%)$, self-employed in oncological joint practice $(n=5$, $45 \%)$ or employed in a hospital $(n=5,45 \%)$ and predominantly did not work in an inpatient PCU $(n=7,63.6 \%)$. Physicians additionally working in inpatient palliative care

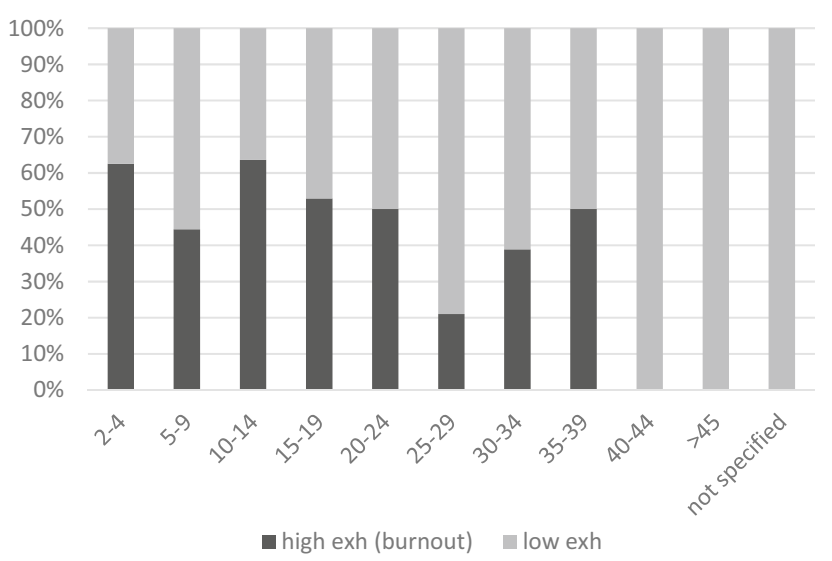

Fig. 2 Professional experience and exhaustion (burnout) 
settings ( $n=4$ ) on average worked $48.25 \% \pm 34.40 \%$ (range $4-81 \%)$ of their time on PCUs.

Pearson's correlation coefficients were calculated to evaluate the correlation between exhaustion and the percentage of engagement in an outpatient palliative care team (SAPV). The results showed a strongly positive correlation $(r=0.607 ; p<0.05)$; that is, the higher the percentage of working time in SAPV was, the higher the level of exhaustion was.

\section{Discussion}

In this study, we assessed the prevalence of burnout among oncologists and its possible relation to different sociodemographic factors. Using stringent diagnostic criteria according to the OLBI, more than $43.8 \%$ of participating German oncologists showed increased exhaustion rates as a sign of burnout. Though this prevalence is lower than the rate of burnout-affected physicians in other disciplines, such as emergency medicine, general internal medicine and neurology (Shanafelt et al. 2012; Peter Chernoff et al. 2019), our data reflect a public health crisis with a huge negative impact on patient care, physicians' health and healthcare organizations and systems (reviewed by West et al. 2018).

The analysis of different healthcare systems showed broad variations regarding the prevalence of burnout among oncologists: Using different diagnostic criteria, even higher rates of burnout were reported in a survey among oncologists in the US (Allegra et al. 2005) or Korea (Lee et al. 2020), whereas a recent survey involving Italian oncologists has only shown a burnout rate of $10.5 \%$ (Cheli et al. 2021).

In this survey, burnout was significantly associated with age and professional experience, with a higher prevalence observed among younger physicians. These data are in line with published results emphasizing the higher risk of burnout among younger physicians at a lower hierarchical level (e.g. residents) (Shanafelt et al. 2012). In contrast, a higher age was associated with a lower prevalence of burnout in this survey, as shown in other surveys involving oncologists in different countries (Alacacioglu et al. 2009; Shanafelt et al. 2014). Higher weekly working hours, higher rates of emotional labor and more alternating shifts, including night or weekend work, might contribute to this increased prevalence (Panagopoulou et al. 2006). Furthermore, working in an inpatient setting and being confronted daily with severely sick patients in an advanced stage of disease might be additional cofactors. Furthermore, the double burden of managing family life and professional career can cause role conflicts as an additional risk factor for burnout (Linzer et al. 2001; Cheli et al. 2021). Interventions to promote the mental health of oncology workers should address these issues.
Several authors (Blanchard et al. 2010; Roth et al. 2011; Shanafelt et al. 2014; Cheli et al. 2021) have proposed a higher prevalence of burnout in female physicians, whereas we and other groups (Wang et al. 2014) could not confirm this observation. However, the number of female physicians participating in this survey was low to draw any conclusions on gender-specific differences.

The decrease in the rate of burnout with advanced age and professional experience probably reflects the capability of physicians to deal with professional requirements and stressors; alternatively, physicians might not work anymore in professional patient care. Moreover, the high level professional role associated with social and financial benefit might be a protective factor against burnout (Cheli et al. 2021).

The work setting of physicians may have a strong impact on professional satisfaction: In contrast to data from the US with higher burnout rates among physicians working in their own private practices than those working in academic medical centers or other practices (Dyrbye et al. 2011, 2013), this survey showed a lower prevalence of exhaustion in self-employed physicians. Given that healthcare systems in Germany are different from those in the US, it can be assumed that physicians in outpatient oncological practices are less confronted with stress factors, such as rotating-shift work or night work. Furthermore, self-employment might allow a better control of the workload leading to less stress and a higher professional satisfaction (Williams et al. 2002). Furthermore, in our cohort, oncologists in outpatient practices tended to be older; thus, the reduced rate of exhaustion might also be attributed to the higher age and the higher grade of experience. Physicians' psychological burden has a strong impact on medical care. In a comprehensive systematic review in 2016, Hall et al. (2016) have reported a high level of evidence for relations between self-reported medical errors and psychological burden. A possible explanation for this relationship is the emergence of cognitive limitations. These findings stress the importance of physicians' health to ensure quality patient care.

Though the rate of exhaustion was high in this survey, the rates of disengagement were lower than expected with a mean value of 1.65 and less than $10 \%$ showing a high rate of disengagement. This prevalence is lower than the prevalence of disengagement of physicians in other disciplines, such as emergency medicine (Chernoff et al. 2019) or medical staff in general. These findings imply high demands of physicians to fulfill job requirements despite imminent or actual overburdening in daily work.

\section{Strengths and limitations}

This study is the first to examine two dimensions of burnout in a German sample of oncologists. We had the opportunity to reach several oncologists through the cooperation 
with the AIO. The prevalence of physicians additionally board certified in palliative care is higher (exceeding 20-30\%) among oncologists than in other medical disciplines (5\% among all medical disciplines in Germany) (KV 2020). This is mainly caused by the fact that patients treated in a palliative setting represent a substantial part of all patients treated in an in- or outpatient setting. As a consequence, aspects of palliative and supportive care are integral part of the daily work among oncologists.

However, this survey has several limitations. First, the number of physicians that responded was limited; physicians with a higher symptom load might have reported at a higher frequency, leading to an overestimation of the prevalence of burnout, though the prevalence of burnout reported here is in line with data published.

Second, since the participants were members of a professional society, the work demands, and job characteristics might not reflect clinical routine in Germany. Third, since this survey was conducted in summer 2020 after the first peak of the coronavirus disease 2019 (COVID-19) pandemic, additional stressors associated with this might have influenced the results of this survey. In contrast, during the survey running, the incidence rates of COVID-19 were low in Germany with only few major structural issues occurring in hospitals. The main limitation of this study is related to its cross-sectional design, which does not allow either temporal or causal inferences. Further investigations with consecutive measurements are required to obtain a more detailed understanding of burnout among oncologists.

\section{Conclusion}

This survey highlights a high rate of exhaustion among German oncologists, with a focus on professional burden during the early years of career. More support is necessary to mitigate potential stressors for medical personnel within the healthcare system. To ensure high-quality medical care, physicians should be offered preventive mental healthcare services early in their careers.

Acknowledgements The authors would like to thank Eva Demerouti for kindly providing the OLBI. The authors would like to thank the participants who made this study possible.

Author contributions MHE: protocol and project development, data collection, data management, first draft of manuscript and manuscript writing. GMH: protocol and project development, data collection, data analysis and manuscript writing. DG: project development and manuscript writing. USB: project development and manuscript writing. IM: protocol and project development, data collection, data analysis and manuscript writing.
Funding Open Access funding enabled and organized by Projekt DEAL. This research did not receive any specific grant from funding agencies in the public, commercial or non-profit sectors.

Availability of data and materials The datasets generated and/or analyzed during this study are available from the corresponding author on reasonable request.

Code availability Not applicable.

\section{Declarations}

Conflict of interest All authors declare no conflicts of interest regarding the publication of this article.

Ethical approval Positive approval was received in September 2019 from the Ethics Committee of the Medical Faculty in Heidelberg (S-615/2019).

Informed consent Informed consent was obtained from the participants through the online version of the survey.

Open Access This article is licensed under a Creative Commons Attribution 4.0 International License, which permits use, sharing, adaptation, distribution and reproduction in any medium or format, as long as you give appropriate credit to the original author(s) and the source, provide a link to the Creative Commons licence, and indicate if changes were made. The images or other third party material in this article are included in the article's Creative Commons licence, unless indicated otherwise in a credit line to the material. If material is not included in the article's Creative Commons licence and your intended use is not permitted by statutory regulation or exceeds the permitted use, you will need to obtain permission directly from the copyright holder. To view a copy of this licence, visit http://creativecommons.org/licenses/by/4.0/.

\section{References}

Alacacioglu A, Yavuzsen T, Dirioz M, Oztop I, Yilmaz U (2009) Burnout in nurses and physicians working at an oncology department. Psychooncology 18:543-548

Allegra CJ, Hall R, Yothers G (2005) Prevalence of burnout in the U.S. Oncology community: results of a 2003 survey. J Oncol Pract $1: 140-147$

Awadh AM, Wan Ismail W (2012) The impact of personality traits and employee work-related attitudes on employee performance with the moderating effect of organizational culture: the case of Saudi Arabia. Asian J Bus Manag Sci 1:108-127

Banerjee S, Califano R, Corral J et al (2017) Professional burnout in European young oncologists: results of the European society for medical oncology (ESMO) young oncologists committee burnout survey. Ann Oncol 28:1590-1596

Bianchi R, Schonfeld IS, Laurent E (2017) Can we trust burnout research? Ann Oncol 28:2320-2321

Blanchard P, Truchot D, Albiges-Sauvin L et al (2010) Prevalence and causes of burnout amongst oncology residents: a comprehensive nationwide cross-sectional study. Eur J Cancer 46:2708-2715

Block RI, Bair HL, Carillo JF (2020) Is exhaustion more sensitive than disengagement to burnout in academic anesthesia? A study using the Oldenburg burnout inventory. Psychol Rep 123:1282-1296 
Bundesärztekammer. (2020). Ärztestatistk zum 31. Dezember 2020. Retrieved from https://www.bundesaerztekammer.de/fileadmin/ user_upload/downloads/pdf-Ordner/Statistik_2020/2020-Statistik. pdf. Accessed 31 Oct 2021

Cheli S, Zagonel V, Oliani C, Blasi L, Fioretto L (2021) Is burnout affecting life satisfaction in oncologists? The moderating role of family concerns in an Italian sample. Psychooncology 30:385-391

Chernoff P, Adedokun C, O'Sullivan I, McManus J, Payne A (2019) Burnout in the emergency department hospital staff at cork university hospital. Ir J Med Sci 188:667-674

Cooper CL, Rout U, Faragher B (1989) Mental health, job satisfaction, and job stress among general practitioners. BMJ 298:366-370

Demerouti E, Demerouti E, Bakker AB, Vardakou I, Kantas A (2003) The convergent validity of two burnout instruments: a multitraitmultimethod analysis. Eur J Psychol Asses 19:12

Demerouti E, Bakker AB (2008) The Oldenburg burnout inventory: a good alternative to measure burnout and engagement. Handbook of stress and burnout in health care: $65-78$

Dewa CS, Loong D, Bonato S, Thanh NX, Jacobs P (2014) How does burnout affect physician productivity? A systematic literature review. BMC Health Serv Res 14:325

Dyrbye LN, Shanafelt TD, Balch CM, Satele D, Sloan J, Freischlag J (2011) Relationship between work-home conflicts and burnout among American surgeons: a comparison by sex. Arch Surg 146:211-217

Dyrbye LN, Varkey P, Boone SL, Satele DV, Sloan JA, Shanafelt TD (2013) Physician satisfaction and burnout at different career stages. Mayo Clin Proc 88:1358-1367

Dyrbye LN, West CP, Satele D, Boone S, Tan L, Sloan J, Shanafelt TD (2014) Burnout among U.S. Medical students, residents, and early career physicians relative to the general U.S. Population Acad Med 89:443-451

Eelen S, Bauwens S, Baillon C, Distelmans W, Jacobs E, Verzelen A (2014) The prevalence of burnout among oncology professionals: oncologists are at risk of developing burnout. Psychooncology 23:1415-1422

Freeborn DK (2001) Satisfaction, commitment, and psychological well-being among HMO physicians. West J Med 174:13-18

Haas JS, Cook EF, Puopolo AL, Burstin HR, Cleary PD, Brennan TA (2000) Is the professional satisfaction of general internists associated with patient satisfaction? J Gen Intern Med 15:122-128

Halbesleben JR, Rathert C (2008) Linking physician burnout and patient outcomes: exploring the dyadic relationship between physicians and patients. Health Care Manage Rev 33:29-39

Hall LH, Johnson J, Watt I, Tsipa A, O'Connor DB (2016) Healthcare staff wellbeing, burnout, and patient safety: a systematic review. PLoS ONE 11:e0159015

Hettiararchchi HAH, Jayarathna SMDY (2014) The effect of employee work related attitudes on employee job performance: a study of tertiary and vocational education sector in Sri Lanka. IOSR JBM $16: 74-83$

IBM, C. (2020) Ibm spss statistics for windows (Version 27.0). Armonk, NY: IBM Corp

Kleiner S, Wallace JE (2017) Oncologist burnout and compassion fatigue: investigating time pressure at work as a predictor and the mediating role of work-family conflict. BMC Health Serv Res $17: 1-8$

KV (2020) Gesundheitsdaten. Retrieved from https://gesundheitsdaten. kbv.de/cms/html/17066.php. Accessed 31 Oct 2021

Lee YG, Maeng CH, Kim DY, Kim BS (2020) Perspectives on professional burnout and occupational stress among medical oncologists: a cross-sectional survey by Korean society for medical oncology (ksmo). Cancer Res Treat 52:1002-1009
Linzer M, Visser MR, Oort FJ et al (2001) Predicting and preventing physician burnout: results from the United States and the Netherlands. Am J Med 111:170-175

Maslach C, Jackson SE, Leiter MP (1981) Maslach burnout inventory: Mbi: consulting psychologists press Palo Alto, CA

Medisauskaite A, Kamau C (2017) Prevalence of oncologists in distress: systematic review and meta-analysis. Psychooncology 26:1732-1740

Oreskovich MR, Kaups KL, Balch CM et al (2012) Prevalence of alcohol use disorders among American surgeons. Arch Surg 147:168-174

Panagopoulou E, Montgomery A, Benos A (2006) Burnout in internal medicine physicians: differences between residents and specialists. Eur J Intern Med 17:195-200

Prins JT, Gazendam-Donofrio SM, Tubben BJ, van der Heijden FM, van de Wiel HB, Hoekstra-Weebers JE (2007) Burnout in medical residents: a review. Med Educ 41:788-800

Rotenstein LS, Torre M, Ramos MA et al (2018) Prevalence of burnout among physicians: a systematic review. JAMA 320:1131-1150

Roth M, Morrone K, Moody K et al (2011) Career burnout among pediatric oncologists. Pediatr Blood Cancer 57:1168-1173

Shanafelt TD, Balch CM, Bechamps G et al (2010) Burnout and medical errors among American surgeons. Ann Surg 251:995-1000

Shanafelt TD, Boone S, Tan L et al (2012) Burnout and satisfaction with work-life balance among us physicians relative to the general US population. Arch Intern Med 172:1377-1385

Shanafelt TD, Gradishar WJ, Kosty M et al (2014) Burnout and career satisfaction among us oncologists. J Clin Oncol 32:678-686

Shanafelt TD, Hasan O, Dyrbye LN et al (2015) Changes in burnout and satisfaction with work-life balance in physicians and the general us working population between 2011 and 2014. Mayo Clin Proc 90:1600-1613

Shanafelt TD, Dyrbye LN, Sinsky C et al (2016a) Relationship between clerical burden and characteristics of the electronic environment with physician burnout and professional satisfaction. Mayo Clin Proc 91:836-848

Shanafelt TD, Mungo M, Schmitgen J, et al (2016b) Longitudinal study evaluating the association between physician burnout and changes in professional work effort. Paper presented at the Mayo Clinic Proceedings.

Sinsky C, Colligan L, Li L, Prgomet M et al (2016) Allocation of physician time in ambulatory practice: a time and motion study in 4 specialties. Ann Intern Med 165:753-760

Trufelli DC, Bensi CG, Garcia JB et al (2008) Burnout in cancer professionals: a systematic review and meta-analysis. Eur J Cancer Care (engl) 17:524-531

van der Heijden F, Dillingh G, Bakker A, Prins J (2008) Suicidal thoughts among medical residents with burnout. Arch Suicide Res 12:344-346

Von Elm E, Altman DG, Egger M et al (2007) The strengthening the reporting of observational studies in epidemiology (strobe) statement: guidelines for reporting observational studies. Bull World Health Organ 85:867-872

Wang Z, Xie Z, Dai J, Zhang L, Huang Y, Chen B (2014) Physician burnout and its associated factors: a cross-sectional study in shanghai. J Occup Health 56:73-83

West CP, Tan AD, Shanafelt TD (2012) Association of resident fatigue and distress with occupational blood and body fluid exposures and motor vehicle incidents. Mayo Clin Proc 87:1138-1144

West CP, Tan AD, Shanafelt TD (2018) Physician burnout: contributors, consequences and solutions. J Intern Med 283:516-529 
Williams ES, Konrad TR, Linzer M et al (2002) Physician, practice, and patient characteristics related to primary care physician physical and mental health: results from the physician worklife study. Health Serv Res 37:121-143

Yates M, Samuel V (2019) Burnout in oncologists and associated factors: a systematic literature review and meta-analysis. Eur J Cancer Care 28:e13094
Publisher's Note Springer Nature remains neutral with regard to jurisdictional claims in published maps and institutional affiliations.* 